\title{
Características bioquímicas de variedades de maíz
}

\author{
Biochemical characteristics of maize varieties \\ Guadalupe Reyes-García $^{1}$ (D) , Francisco Palemón-Alberto ${ }^{1 *}$ (D) , Noel Orlando Gómez-Montiel² (iD , \\ Alejandro Espinosa-Calderón ${ }^{3}$ (D), Santo Ángel Ortega-Acosta ${ }^{1}$ (D) , Fernando Castillo- \\ González ${ }^{4}$ (D) , Alfredo Josué Gámez-Vázquez ${ }^{5}$ (D) , Delia Moreno-Velázquez ${ }^{6}$ (D) , \\ Cesar del Ángel Hernández-Galeno² (D), Agustín Damián-Nava ${ }^{1}$ (D)

\begin{abstract}
${ }^{1}$ Facultad de Ciencias Agropecuarias y Ambientales, Universidad Autónoma de Guerrero, Periférico poniente s/n, Colonia Villa de Guadalupe, 40010, Iguala de la Independencia, Guerrero, México.

${ }^{2}$ Campo Experimental Iguala-Instituto Nacional de Investigaciones Forestales, Agrícolas y Pecuarias (INIFAP), Carretera Iguala-Tuxpan km 2.5, 40000, Iguala de la Independencia, Guerrero, México.

${ }^{3}$ Campo Experimental Valle de México-INIFAP, Carretera Los Reyes-Texcoco km 13.5, 56250, Coatlinchán, Texcoco, Estado de México, México.

${ }^{4}$ Colegio de Postgraduados, Carretera México-Texcoco km 36.5, 56230, Montecillo, Texcoco, Estado de México, México.

${ }^{5}$ Campo Experimental Bajío-INIFAP, Carretera Celaya-San Miguel de Allende km 6.5, 38110, Celaya, Guanajuato, México.

${ }^{6}$ Facultad de Ingeniería Agrohidráulica, Benemérita Universidad Autónoma de Puebla, Av. Universidad s/n Junta Auxiliar de San Juan Acateno, Teziutlán, Puebla, 73695, México.
\end{abstract} \\ *Autor para correspondencia: alpaf75@hotmail.com
}

Fecha de recepción:

24 de junio de 2020

Fecha de aceptación:

8 de agosto de 2020

Disponible en línea:

19 de junio de 2021

Este es un artículo en acceso abierto que se distribuye de acuerdo a los términos de la licencia Creative Commons.

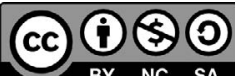

Reconocimiento-

NoComercia-

CompartirIgual 4.0

Internacional

\section{RESUMEN}

El maíz es importante como grano básico, por ser la principal fuente de alimento del pueblo mexicano. En el estado de Guerrero, existe una gran diversidad de regiones agroecológicas, y los agricultores siembran bajo condiciones de riego y temporal. La siembra de maíz en las comunidades rurales se lleva a cabo principalmente en forma manual y aplican nitrógeno como fertilizante en dos etapas fenológicas del cultivo. El objetivo de la presente investigación fue conocer el contenido de proteína y el porcentaje de dos aminoácidos esenciales presentes en el maíz, al aplicar diversas dosis de nitrógeno y fósforo en tres variedades utilizadas en la región. El experimento se estableció en las localidades de Iguala y Apaxtla, con un diseño experimental de bloques completos al azar y tres repeticiones en arreglo factorial. La unidad experimental fue de cuatro surcos de $5 \mathrm{~m}$ de longitud y $0.81 \mathrm{~m}$ entre surcos. Para la fertilización, se utilizaron el nitrógeno y el fósforo como factores . Para cada tratamiento, se estimó el rendimiento de grano, el porcentaje de proteína, triptófano y lisina. Los resultados indicaron que los tratamientos exhibieron un efecto significativo en las cuatro variables evaluadas en los genotipos de maíz.

\section{PALABRAS CLAVE}

Zea mays, nitrógeno, fósforo, rendimiento de grano

\section{ABSTRACT}

Corn is important as a basic grain, as it is the main food source for people in Mexico. There is a great diversity of agroecological regions in Guerrero, where farmers plant under irrigation and temporary conditions. In rural communities, corn is sown mainly by hand, and nitrogen is usually applied as a fertilizer in two phenological stages of the crop. The objective of this study was to know the content of protein and the percentage of two essential amino acids in corn when applying nitrogen and phosphorus in different doses to three corn varieties of this region. The experiment was carried out in Iguala and Apaxtla, Guerrero, with an experimental design of complete blocks at random with three repetitions in a factorial arrangement. The experimental unit was made up of four five-meter-long furrows with $0.81 \mathrm{~m}$ between each of them. For the fertilization, nitrogen and 
phosphorus were used as factors. The yield of grain, the percentage of protein, tryptophan, and lysine were estimated. The results indicated that the treatments exhibited a significant effect on the four variables measured in the genotypes.

KEYWORDS

Zea mays, nitrogen, phosphorus, grain yield.

\section{INTRODUCCIÓN}

México es el centro de diversidad genética del maíz (Zea mays L.), cultivo de gran importancia económica y básico para la alimentación humana. La gran diversidad del maíz difiere en su composición química, propiedades y utilización final. En el estado de Guerrero, existen diversas regiones agroecológicas (Palemón-Alberto et al. 2011), donde el maíz se cultiva bajo condiciones de riego y temporal. En las comunidades rurales, los agricultores generalmente aplican fertilizante nitrogenado en dos etapas fenológicas del cultivo, y la siembra se realiza en suelos de lomerío y ladera, donde los rendimientos de grano oscilaron entre 6.3 a $2.5 \mathrm{t}$ ha-1 (Palemón-Alberto et al. 2016; Palemón-Alberto et al. 2017).

Los agricultores han sembrado genotipos de maíz como: la variedad sintética VS-535 (elote, forraje y grano), el híbrido trilineal A-7573 (elote) o la variedad V-537C con calidad de proteína (grano), mismas que se han adaptado a las condiciones ambientales de la región. Estas variedades son una alternativa para fortalecer a los agricultores y que éstos puedan obtener rendimientos de grano superiores a las $2.5 \mathrm{t}$ ha- 1 en suelos de lomerío (Gómez et al. 2003).

Para incrementar la producción de grano de maíz, es importante conocer las funciones del nitrógeno y del fósforo como nutrimentos esenciales para el crecimiento y desarrollo de dicho cultivo. Diversos estudios indican que el nitrógeno desempeña un papel importante para la producción de clorofila, enzimas, proteínas y ácidos nucleicos en los cereales (Adediran y Banjoko 1995). Cabe señalar que éste se moviliza a través del tallo y de las hojas (Ciampitti y Vyn 2013). El suministro adecuado de nitrógeno asegura un buen rendimiento de grano de maíz, principalmente en el incremento de número de granos (Uribelarrea et al. 2004; Khaliq et al. 2009), además de mejorar la calidad de forraje y el contenido de proteínas (Haque et al. 2001). Acorde con algunos autores, la aplicación de dosis altas de nitrógeno incrementa la altura de la planta, el diámetro del tallo (Sotomayor et al. 2017), así como el rendimiento del grano (Chura et al. 2019), la proteína y la lisina (Barrios y Basso 2018).

Por su parte, el fósforo contribuye a la formación de los ácidos nucleicos, así como en la respiración celular y la actividad metabólica; asimismo, al aplicarse con el nitrógeno, aumentan el rendimiento de grano, la calidad de forraje, la altura de planta y el número de hojas por planta (Masood et al. 2011).

En maíz, se ha cuantificado el contenido de minerales (Menkir 2008), proteína, lisina y triptófano (Zepeda-Bautista et al. 2009; Vera-Guzmán et al. 2012), aceite (Torres-Morales et al. 2010), biosíntesis de almidón (Agama-Acevedo et al. 2013), provitamina A (Owens et al. 2014; Pillay et al. 2014), entre otras sustancias. Así, de acuerdo con los antecedentes, como objetivo, se planteó conocer el contenido de proteína y el porcentaje de dos aminoácidos esenciales presentes en maíz cultivado en la región Norte del estado de Guerrero, México, al aplicar diversas dosis de nitrógeno y fosforo; además, como hipótesis se proyectó que el efecto del ambiente y la fertilización modificarían el porcentaje de proteína, lisina y triptófano en las variedades de maíz.

\section{Materiales y Métodos}

\section{Ubicación del sitio de estudio}

El experimento se estableció en las localidades de Apaxtla ( $\left(18^{\circ} 08^{\prime} 1^{\prime \prime} \mathrm{N}, 99^{\circ} 55^{\prime} 79^{\prime \prime} \mathrm{O}, 1,182 \mathrm{msnm}\right)$ e Iguala

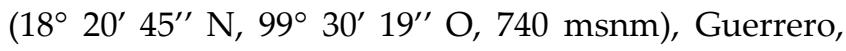
México, en el ciclo agrícola primavera-verano de 2018. El clima de las localidades es tipo Awo (w) (i) g y A(C) w2(w)ig, con precipitación pluvial promedio en verano de 977 y 1,100 mm (García 2004). 
Material biológico. Los genotipos evaluados fueron: variedad sintética VS-535, variedad de polinización libre V-537C e híbrido trilineal A-7573 (Monsanto-Asgrow). Las variedades son genotipos liberados por el Campo Experimental Iguala del Instituto Nacional de Investigaciones Forestales, Agrícolas y Pecuarias (INIFAP), VS-535 en 1993 y V-537 C en 2000. La variedad sintética VS-535 está conformada con germoplasma tuxpeño y se caracteriza por su mayor producción de elote, forraje y grano (Gómez et al. 2016); la V-537C (maíz con calidad de proteína (QPM)), proveniente de la población Poza Rica 8763 y conformada por germoplasma de la raza tuxpeño, grano semidentado, se adapta a regiones de clima cálido húmedo y subhúmedo, con altitud inferior a 1,200 m (Gómez et al. 2003). Por último, A-7573 es un híbrido comercial, caracterizado por ser elotero (Sánchez et al. 2013).

\section{Diseño experimental}

El diseño experimental utilizado fue de bloques completos al azar con arreglo factorial y tres repeticiones. Los factores evaluados fueron: genotipos (VS-535, V-537-C y A-7573); dosis de nitrógeno (100, 150 y $\left.200 \mathrm{~kg} \mathrm{ha}^{-1} \mathrm{~N}\right)$ y dosis de fósforo $\left(60,90\right.$ y $120 \mathrm{~kg} \mathrm{ha}^{-1}$ $\mathrm{P})$. La unidad experimental constó de cuatro surcos de $5 \mathrm{~m}$ de longitud y $0.81 \mathrm{~m}$ de ancho. Los experimentos se condujeron en ambas localidades bajo condiciones de temporal (secano). Las fuentes de fertilizante fueron: nitrógeno (Urea 46\%) y fósforo (fosfato diamónico), las aplicaciones se efectuaron a los 25, 40 y 55 días después de la siembra (dds) para el caso del N, y se aplicó todo el $\mathrm{P}$ a los 25 dds.

\section{Variables analizadas}

En campo, el rendimiento de grano (RGr $\left.t \mathrm{ha}^{-1}\right)$ fue determinado con la fórmula:

\section{$\mathrm{RGr}=\mathrm{PDC}^{*} \mathrm{FDD}^{*} \mathrm{FDH}^{*} \mathrm{FCUS}$,}

donde; PDC: peso de campo; FDD: factor de desgrane; FDH: factor de humedad; FCUS: factor de corrección por unidad de superficie, ajustado a 12 por ciento de humedad para ambas localidades (Palemón-Alberto et al. 2017).
El rendimiento se estimó de la siguiente manera:

PDC $x$ FDD $x$ FDH $x$ FCUS a Kg ha-1. Donde, FCUS $=10,000 \mathrm{~m}^{2} /$ área de la parcela.

En laboratorio, se determinó el contenido de proteína, lisina y triptófano. El porcentaje de proteína $(\mathrm{P} \%)$ se obtuvo de la siguiente manera: se pesaron 100 $\mathrm{g}$ de fenolftaleína y se disolvió en alcohol etílico en un matraz volumétrico de $100 \mathrm{~mL}$; asimismo, se pesaron $33 \mathrm{mg}$ de bromo crisol verde y $66 \mathrm{mg}$ de metilo rojo y se depositaron en un matraz volumétrico de $100 \mathrm{~mL}$. Además, se disolvieron $10 \mathrm{mg}$ de ácido bórico en 1,400 $\mathrm{mL}$ de agua destilada, $70 \mathrm{~mL}$ de solución indicadora "a" y $20 \mathrm{~mL}$ de solución "b", lo cual se aforó a 2,000 mL con agua destilada y se ajustó el color a café rojizo con sosa diluida $(0.1 \mathrm{~N})$. La proteína y la materia orgánica fueron oxidadas por el ácido sulfúrico, mientras que el nitrógeno presente en forma orgánica se fijó como sulfato de amonio, pues al hacer reaccionar esta sal con una base fuerte se desprende el amoniaco. Por titulación del ácido no neutralizado se calculó la cantidad de amoniaco desprendido y el nitrógeno $(\mathrm{N})$ en la muestra. El porcentaje (\%) de $\mathrm{N}$ multiplicado por 6.25 dio como resultado el porcentaje de proteína.

Por otra parte, se pesaron muestras de $10 \mathrm{mg}$ por triplicado, mismas a las que se les adicionó $2.5 \mathrm{~mL}$ de P-DMAB y $12 \mathrm{~mL}$ de ácido sulfúrico a 60 por ciento, hasta lograr el volumen de $14.5 \mathrm{~mL}$; se agitaron y se sometieron a oscuridad durante $17 \mathrm{~h}$; posteriormente, se adicionó $0.1 \mathrm{~mL}$ de nitrito de sodio al 0.05 por ciento, se dejó reposar por 30 minutos y se registraron las lecturas en un espectrofotómetro (UV-VIS, Shimadzu Scientific Instruments, Inc.), a $590 \mathrm{~nm}$, con apoyo de testigo. Los datos obtenidos se compararon con la curva de calibración estándar, para cotejar el contenido de triptófano (T\%) presente en la muestra.

Para determinar el contenido de lisina (L\%), se pesaron $2 \mathrm{mg}$ de muestra finamente molida en dos frascos (A, B); al frasco A, se le agregaron $15 \mathrm{mg}$ de lisina, arginina, basidina, $1 \mathrm{~mL}$ de 2-propanol, y se agitó durante 5 minutos para humedecer ambas muestras, luego de lo cual se agregaron $4 \mathrm{~mL}$ de acetato de sodio trihidratado a 5 por ciento; por su parte, al frasco B se le agregaron $0.3 \mathrm{~mL}$ de anhídrido propiónico, se agitó durante 15 minutos y posteriormente se le agregaron $40 \mathrm{~mL}$ de solución con colorante y se agitó en una 
centrifuga (Marca Beckman, Modelo Avanti-J-25, Beckman instruments, Inc.), durante 60 minutos, hasta alcanzar el equilibrio para centrifugarse a 5,000 rpm durante 10 minutos. Se prepararon $1.36 \mathrm{~g}$ de colorante en un litro de solución reguladora (20 g de ácido oxálico, $3.4 \mathrm{~g}$ de fosfato monobásico de potasio); además, se le agregaron $60 \mathrm{~mL}$ de ácido acético glacial y se diluyó en un litro de agua. La concentración final del colorante estuvo en un intervalo de 1.3 a $1.7 \mathrm{mmol}$ L y se determinó por espectrometría a $7 \mathrm{~nm}$ (UV-VIS, Shimadzu Scientific Instruments, Inc.). La cantidad de lisina se calculó mediante la siguiente fórmula: moles de colorante ligado ${ }^{*} 16 \mathrm{~g}$ de $\mathrm{N}^{*} 0.146$ (factor de conversión para lisina); el resultado se expresó en $\mathrm{g}$ de lisina / $16 \mathrm{~g}$ de nitrógeno y se reportó en porcentaje.

Análisis estadístico. Se efectuó el análisis de varianza combinado de localidades y comparación de medias con la prueba de Tukey ( $\mathrm{p} \leq 0.01,0.05$ ); para dicho análisis estadístico se utilizó el programa Statistical Analysis Systems (SAS) Versión 9.0.

\section{Resultados Y Discusión}

\section{Factores y variables de estudio}

Para el rendimiento de grano (RGr) no se detectaron diferencias estadísticas significativas en la fuente de variación localidades (L). Por el contrario, en tratamientos $(\mathrm{T})$, genotipos $(\mathrm{G})$, nitrógeno $(\mathrm{N})$, e interacción $\mathrm{G} \times$ fósforo $(\mathrm{P})$ y $\mathrm{N} \times \mathrm{P}$ sí se observaron diferencias significativas $(p \leq 0.01)$. En la interacción $\mathrm{L} \times \mathrm{G}, \mathrm{L} \times \mathrm{N}$ y $\mathrm{L} \times \mathrm{P}$, no se detectaron efectos significativos en la misma variable (Cuadro 1 ).

En la respuesta del material genético respecto al contenido de proteína, se observaron diferencias significativas en las 17 fuentes de variación; sin embargo, por la dimensión de sus cuadrados medios, lo que más influyó en esta característica fue la localidad de evaluación. Este resultado indica la existencia de variabilidad entre los genotipos en cada ambiente (Manjarrez et al. 2014). En triptófano y lisina, a pesar de haber sido significativas algunas fuentes de variación e interacciones, por la magnitud de sus cuadrados medios, la dosis de nitrógeno tuvo mayor injerencia en la manifestación de estas dos características. Estos resultados sugieren que, al evaluar los genotipos de un ambiente a otro, expresaron de manera diferencial el porcentaje de proteína; en cambio, en el contenido de triptófano y lisina de los genotipos VS-535, A-7573 y V-537C, no hubo efecto de los tratamientos al evaluarse en dos ambientes (Cuadro 1). Es importante mencionar que el rendimiento de grano está influenciado por muchos genes (ZmDA1, ZmPLA1, o2, wx, olc1, ln1, su1) (Xie et al. 2018; Zhang et al. 2013) y por las condiciones donde fueron expuestas para expresar su máximo potencial.

\section{Localidades}

El rendimiento de grano $\left(\mathrm{t} \mathrm{ha}^{-1}\right)$ promedio observado en ambas localidades fue estadísticamente similar, aunque en la localidad de Apaxtla se lograron $288 \mathrm{~kg}$ más respecto a Iguala. Este resultado significa que el comportamiento de los tres genotipos no varió significativamente en las dos localidades, debido a que el material genético ha sido seleccionado y adaptado en condicionesambientalessemejantes(Gómezetal.2003). En Apaxtla, el porcentaje de proteína fue superior estadísticamente $(10.343 \%)$, respecto a la localidad de Iguala (9.318\%); es decir, hubo una diferencia significativa de 1.025 por ciento de proteína (Cuadro 2).

Los porcentajes promedio de triptófano $\mathrm{y}$ lisina fueron similares estadísticamente en ambas localidades (Cuadro 2). Los resultados indican que no hubo cambios significativos de ambos aminoácidos extraídos en el grano de los tres genotipos, por lo que es importante evaluar la estabilidad de los genotipos en un mayor número de ambientes, para valorar características agronómicas y componentes de rendimiento (Palemón-Alberto et al. 2012a).

\section{Tratamientos}

En el factor tratamientos, para la variable rendimiento de grano, el tratamiento nueve (VS-535 + 200 N + 120 $\mathrm{P})$, presentó mayor valor (7.1 t ha-1) y superó estadísticamente a seis tratamientos (VS-535 + $100 \mathrm{~N}+90$ $\mathrm{P}, \mathrm{VS}-535+150 \mathrm{~N}+120 \mathrm{P}, \mathrm{A}-7573+100 \mathrm{~N}+60 \mathrm{P}, \mathrm{A}-7573$ $+100 \mathrm{~N}+120 \mathrm{P}, \mathrm{A}-7573+150 \mathrm{~N}+120 \mathrm{P}, \mathrm{A}-7573+200$ $\mathrm{N}+120 \mathrm{P}$ ); adicionalmente, se observaron valores inferiores en trece tratamientos comparados con el 
Cuadro 1. Cuadrados medios y significancia estadística de los análisis de varianza en la evaluación de tres genotipos de maíz cultivados en dos ambientes bajo tratamientos de $\mathrm{N}$ y $\mathrm{P}$, para cuatro variables cuantificadas en laboratorio.

\begin{tabular}{|c|c|c|c|c|c|}
\hline \multirow[b]{2}{*}{$\mathrm{FV}$} & \multicolumn{4}{|c|}{ Cuadrados medios } & \multirow[b]{2}{*}{ Lisina $(\%)$} \\
\hline & GL & RGr $\left(\mathrm{t} \mathrm{ha}^{-1}\right)$ & Proteína $(\%)$ & Triptófano (\%) & \\
\hline Localidades (L) & 1 & 3.38 & $42.56^{* *}$ & 0.02 & 2.77 \\
\hline Repeticiones & 2 & 6.79 & 0.06 & 0.11 & 5.86 \\
\hline Tratamientos $(\mathrm{T})$ & 26 & $3.77^{* *}$ & $4.11^{* *}$ & $0.28^{* *}$ & $9.95 *$ \\
\hline $\mathrm{L} \times \mathrm{T}$ & 26 & 0.32 & $6.20 * *$ & $0.16^{* *}$ & 6.35 \\
\hline Genotipos (G) & 2 & $9.09 * *$ & $2.92^{* *}$ & 0.15 & 10.21 \\
\hline Nitrógeno $(\mathrm{N})$ & 2 & $9.01 * *$ & $4.19^{* *}$ & $0.78^{* *}$ & $22.80 *$ \\
\hline Fósforo (P) & 2 & 1.71 & $8.63^{* *}$ & $0.39 * *$ & 15.30 \\
\hline $\mathrm{G} \times \mathrm{N}$ & 4 & 0.51 & $4.87^{* *}$ & 0.15 & 5.80 \\
\hline$G \times P$ & 4 & $5.39 * *$ & $3.75^{* *}$ & $0.25^{*}$ & 7.80 \\
\hline $\mathrm{N} \times \mathrm{P}$ & 4 & $5.39 * *$ & $4.40^{* *}$ & $0.22 *$ & 10.31 \\
\hline $\mathrm{G} \times \mathrm{N} \times \mathrm{P}$ & 8 & 1.64 & $3.41 * *$ & $0.26^{* *}$ & 8.29 \\
\hline $\mathrm{L} \times \mathrm{G}$ & 2 & 0.24 & $15.44^{* *}$ & 0.11 & 15.03 \\
\hline $\mathrm{L} \times \mathrm{N}$ & 2 & 0.45 & $13.05^{* *}$ & 0.19 & 7.92 \\
\hline $\mathrm{L} \times \mathrm{P}$ & 2 & 0.09 & $9.25 * *$ & $0.38^{* *}$ & 2.55 \\
\hline $\mathrm{L} \times \mathrm{G} \times \mathrm{N}$ & 4 & 0.51 & $2.18^{* *}$ & 0.17 & $13.37^{*}$ \\
\hline$L \times G \times P$ & 4 & 0.22 & $3.79 * *$ & 0.04 & 3.09 \\
\hline $\mathrm{L} \times \mathrm{N} \times \mathrm{P}$ & 4 & 0.19 & $2.68 * *$ & 0.12 & 7.88 \\
\hline $\mathrm{L} \times \mathrm{G} \times \mathrm{N} \times \mathrm{P}$ & 8 & 0.39 & $6.39 * *$ & $0.19^{* *}$ & 2.08 \\
\hline Error & 132 & 0.99 & 1.30 & 0.09 & 5.79 \\
\hline Media $=$ & & 5.41 & 5.41 & 1.28 & 4.61 \\
\hline $\mathrm{CV}(\%)=$ & & 18.42 & 18.42 & 23.49 & 52.19 \\
\hline
\end{tabular}

FV: Fuente de variación; L: Localidades; T: Tratamientos; G: Genotipos; N: Nitrógeno; P: Fósforo; GL: Grados de libertad; RGr: Rendimiento de grano; CV (\%): Coeficiente de variación en porcentaje; ${ }^{*} \mathrm{y} *$ : Significativo y Altamente significativo al 0.01 y $0.05 \%$.

Cuadro 2. Comparación de medias entre localidades (Iguala y Apaxtla, Guerrero, México) para cuatro variables de tres genotipos de maíz bajo tratamientos de fertilizantes.

\begin{tabular}{lllll}
\hline & \multicolumn{2}{l}{ Localidades } & & \\
\cline { 2 - 3 } Variables & Iguala & Apaxtla & Media & DSH \\
\hline Rendimiento de grano (t ha-1) & $5.261 \mathrm{a}$ & $5.549 \mathrm{a}$ & 5.41 & 1.007 \\
Proteína (\%) & $9.318 \mathrm{~b}$ & $10.343 \mathrm{a}$ & 9.83 & 0.105 \\
Triptófano (\%) & $1.293 \mathrm{a}$ & $1.269 \mathrm{a}$ & 1.28 & 0.094 \\
Lisina (\%) & $4.741 \mathrm{a}$ & $4.479 \mathrm{a}$ & 4.61 & 0.105 \\
\hline
\end{tabular}

DSH: diferencia significativa honesta (Tukey $=\alpha=\mathrm{p} \leq 0.05$ ); variables con la misma letra sobre filas son iguales estadísticamente.

promedio general (5.4), respectivamente (Cuadro 3).

En trabajos previos, la variedad sintética VS-535, evaluada en la región Montaña (localidad Olinalá), región Norte (localidad Teloloapan), expresó $4.377 \mathrm{t}_{\text {ha }}{ }^{-1}$ de rendimiento de grano con el tratamiento 120N+90P (Palemón-Alberto et al. 2012b; Palemón-Alberto et al. 2016; Palemón-Alberto et al. 2017). Mukhtar et al. (2011) reportaron que, al incrementar los niveles de nitrógeno y fósforo (300 $\mathrm{N}+150$ P), disminuyó el rendimiento de grano. 
Cuadro 3. Comparación de medias de 27 tratamientos de fertilización, aplicados en tres genotipos de maíz y evaluados en Iguala y Apaxtla, Guerrero, México.

\begin{tabular}{|c|c|c|c|c|}
\hline Tratamientos & RGr (t ha-1) & Proteína $(\%)$ & Triptofano (\%) & Lisina $(\%)$ \\
\hline 1. VS-535+100N+60P & 5.3abcd & $10.5 \mathrm{bcd}$ & 1.36abcd & 5.98abcd \\
\hline 2. VS- $535+100 N+90 P$ & $4.6 \mathrm{bcd}$ & $8.3 \mathrm{j}$ & $1.85 \mathrm{abcd}$ & $3.29 \mathrm{abcd}$ \\
\hline 3. VS- $535+100 \mathrm{~N}+120 \mathrm{P}$ & 5.3abcd & 9.9cdefg & $1.28 \mathrm{abcd}$ & $8.42 \mathrm{abcd}$ \\
\hline 4. VS- $535+150 N+60 P$ & 5.1abcd & 11.2abcd & $1.13 \mathrm{~cd}$ & $4.24 \mathrm{abcd}$ \\
\hline 5. VS- $535+150 N+90 P$ & 6.0abcd & 10.1cdef & $1.18 \mathrm{bcd}$ & $5.35 \mathrm{abcd}$ \\
\hline 6. VS-535+150N+120P & $4.5 \mathrm{bcd}$ & $10.2 \mathrm{~cd}$ & 1.15abcd & 3.46abcd \\
\hline 7. VS- $535+200 N+60 P$ & 6.1abcd & 9.7defhgi & $1.28 \mathrm{abcd}$ & $3.18 \mathrm{bcd}$ \\
\hline 8. VS- $535+200 N+90 P$ & $4.8 \mathrm{abcd}$ & 11.3abcd & $1.27 \mathrm{abcd}$ & $2.75 \mathrm{bcd}$ \\
\hline 9. VS-535+200N+120P & 7.1abcd & 9.4fghi & 1.34abcd & 3.99abcd \\
\hline 10. $A-7573+100 N+60 P$ & $4.8 \mathrm{bcd}$ & 9.5efghi & $1.38 \mathrm{abcd}$ & 7.08abcd \\
\hline 11. A-7573+100N+90P & 5.1abcd & $9.2 \mathrm{hi}$ & 1.33abcd & $4.16 \mathrm{abcd}$ \\
\hline 12. $\mathrm{A}-7573+100 \mathrm{~N}+120 \mathrm{P}$ & $3.9 \mathrm{~d}$ & 10.0cdefg & $1.19 \mathrm{bcd}$ & $5.02 \mathrm{abcd}$ \\
\hline 13. $A-7573+150 N+60 P$ & 5.4abcd & $10.2 \mathrm{~cd}$ & $1.26 \mathrm{bcd}$ & 6.67abcd \\
\hline 14. $\mathrm{A}-7573+150 \mathrm{~N}+90 \mathrm{P}$ & 5.9abcd & 9.6defghi & $1.37 \mathrm{abcd}$ & $5.02 \mathrm{abcd}$ \\
\hline 15. A-7573+150N+120P & $4.1 \mathrm{~cd}$ & $10.2 \mathrm{~cd}$ & $1.14 \mathrm{~cd}$ & 3.44abcd \\
\hline 16. $A-7573+200 N+60 P$ & 6.1abcd & 9.1hi & $1.06 \mathrm{~cd}$ & $4.95 \mathrm{abcd}$ \\
\hline 17. A-7573+200N+90P & 5.4abcd & 9.1hi & $0.84 \mathrm{~d}$ & 4.37abcd \\
\hline 18. A-7573+200N+120P & $4.3 \mathrm{bcd}$ & 9.4ghi & 1.41abcd & $5.06 \mathrm{abcd}$ \\
\hline 19. $V-537 C+100 N+60 P$ & 4.7abcd & $10.5 \mathrm{bcd}$ & 1.32abcd & $4.36 \mathrm{abcd}$ \\
\hline 20. $V-537 C+100 N+90 P$ & 5.6abcd & $8.1 \mathrm{j}$ & $1.76 \mathrm{abcd}$ & $4.96 \mathrm{abcd}$ \\
\hline 21. $V-537 C+100 N+120 P$ & 5.9abcd & 9.7defgh & $1.30 \mathrm{abcd}$ & $4.53 \mathrm{abcd}$ \\
\hline 22. $\mathrm{V}-537 \mathrm{C}+150 \mathrm{~N}+60 \mathrm{P}$ & 6.0abcd & 9.3ghi & 1.42abcd & $4.75 \mathrm{abcd}$ \\
\hline 23. $V-537 C+150 N+90 P$ & 6.2abcd & $9.0 \mathrm{i}$ & $1.24 \mathrm{bcd}$ & $3.55 \mathrm{abcd}$ \\
\hline 24. $V-537 C+150 N+120 P$ & 5.0abcd & 10.1cde & $1.19 \mathrm{bcd}$ & 3.92abcd \\
\hline 25. $V-537 C+200 N+60 P$ & 5.9abcd & $10.2 \mathrm{~cd}$ & $1.08 \mathrm{~cd}$ & $4.88 \mathrm{abcd}$ \\
\hline 26. $V-537 C+200 N+90 P$ & 6.3abcd & 9.5efghi & $1.55 \mathrm{abcd}$ & $3.11 \mathrm{bcd}$ \\
\hline 27. V-537C+200N+120P & 6.6abcd & 11.8abcd & $0.91 \mathrm{~d}$ & $3.95 \mathrm{abcd}$ \\
\hline Media $=$ & 5.40 & 9.83 & 1.28 & 4.610 \\
\hline $\mathrm{DSH}=$ & 2.30 & 0.700 & 0.589 & 5.172 \\
\hline
\end{tabular}

RGr: Rendimiento de grano; variables con la misma letra son iguales estadísticamente; DSH: diferencia significativa honesta (Tukey, p $\leq$ 0.05).

El porcentaje de proteína (11\%) fue similar estadísticamente en tres tratamientos. Los valores inferiores fueron representados por los tratamientos VS-535+100 N+90 P y $\quad$ V-537C+100 N+90 P. El tratamiento VS-535 + $100 \mathrm{~N}+90 \mathrm{P}$ presentó mayor porcentaje de triptófano (1.85\%); en cambio, los valores inferiores fueron observados en los tratamientos A-7573 + $200 \mathrm{~N}+90 \mathrm{P}$ y V-537C $+200 \mathrm{~N}+120 \mathrm{P}$, con diferencias de 1.01 y 0.94 por ciento respecto al mayor valor $(1.85 \%)$, respectivamente (Cuadro 3 ).

El mayor porcentaje de lisina fue observada en el tratamiento VS-535 + $100 \mathrm{~N}+120 \mathrm{P}$; mientras que los tratamientos VS-535 + $200 \mathrm{~N}+90$ P y VS-537C + $200 \mathrm{~N}$ + $90 \mathrm{P}$, estadísticamente, exhibieron menor porcentaje de lisina ( 2.75 y $3.11 \%$ ). Los resultados indican que los tratamientos fueron eficientes para marcar diferencias significativas en rendimiento de grano, porcentaje de proteína, triptófano y lisina. Adicionalmente, se puede mencionar que cada variable responde con un tratamiento específico de fertilización.

Genotipos. Las variedades VS-535 y V-537C presentaron rendimiento de grano similar en ambas localidades, y A-7573 produjo $820 \mathrm{~kg}$ menos de grano en promedio, lo cual es relevante, pues las dos primeras 
son variedades y A-7573 es un híbrido trilineal (Cuadro 4). Resultados similares fueron observados en la VS-535, al evaluarse en las localidades de Olinalá y Teloloapan (Palemón-Alberto et al. 2012b). Ortiz-Torres et al. (2013) evaluaron 16 poblaciones nativas de maíz y usaron como testigo el híbrido A-7573; estos autores reportaron en promedio $4.073 \mathrm{t}$ ha- 1 de $\mathrm{RGr}$, valor inferior (5.41 t ha-1) al observado en este estudio. La variedad V-537C produjo en promedio $4.06 \mathrm{t}$ ha-1 en cuatro localidades (Andrés-Meza et al. 2014), en el presente estudio; el RGr obtenido fue superior en 349 kg más de producción.

Los genotipos difirieron en el porcentaje de proteína: la variedad sintética VS-535 fue la que presentó mayor valor, seguida de la variedad mejorada V-537C y del híbrido A-7573, con el porcentaje menor de proteína, estadísticamente. Los investigadores han señalado que la calidad de proteína en maíz es un carácter recesivo (Mertz et al. 1964; Crow y Kermicle 2002), y se atribuye al hecho de que la variedad V-537C fue polinizada por A-7573 y VS-535, debido a que coincidieron las floraciones de los genotipos. En este sentido, enmascararon la expresión real del porcentaje de lisina y triptófano, debido a que el endospermo normal fue dominante al gen opaco-2 y, consecuentemente, tuvo una expresión normal la variedad V-537C. En un estudio efectuado sobre composición de fertilización, para estudiar la composición química, y reportaron 4.9 por ciento de lisina y 2.4 por ciento de triptófano, resultados semejantes a los observados en esta investigación.

\section{Nitrógeno}

El factor de variación nitrógeno $(\mathrm{N})$ afectó significativamente la respuesta de los genotipos (Mukhtar et al. 2011). En otros trabajos de investigación realizados en maíz, se ha reportado que, al aplicar 200 unidades de $\mathrm{N}$, se incrementó el número de estigmas (Ciampitti y Vyn 2013; Beres et al. 2012; Ciampitti et al. 2012). Kovács et al. (2014) aplicaron 145 unidades de N y reportaron un incremento en rendimiento de grano. Otros investigadores señalaron que la dosis óptima de nitrógeno influye en el índice de área foliar, en el rendimiento de grano de maíz (Ding et al. 2005) y en el contenido de clorofila (Majnooni-Heris et al. 2011); además, Liu y Zhang (2007) indicaron que debe haber humedad disponible en el suelo para obtener mayor producción de grano. Para poder valorar los porcentajes de proteína, triptófano y lisina en maíz, a priori, la planta de maíz debió pasar por diferentes etapas fenológicas (Chura et al. 2019), en las cuales es importante tomar en cuenta el índice de área foliar y el contenido de clorofila (Iglesias et al. 2018), las

Cuadro 4. Comparación de medias de tres genotipos de maíz, para las variables rendimiento de grano, proteína (\%), triptófano (\%), y lisina (\%); se considera el promedio de dos ambientes y tratamientos de $\mathrm{N}$ y $\mathrm{P}$.

\begin{tabular}{|c|c|c|c|c|c|}
\hline \multirow[b]{2}{*}{ Variables } & \multicolumn{3}{|c|}{ Genotipos } & \multirow[b]{2}{*}{ Media } & \multirow[b]{2}{*}{ DSH (0.05) } \\
\hline & VS-535 & A-7573 & V-537C & & \\
\hline Rendimiento de grano $\left(\mathrm{t} \mathrm{ha}^{-1}\right)$ & $5.417 \mathrm{a}$ & $4.989 \mathrm{~b}$ & $5.809 \mathrm{a}$ & 5.41 & 0.480 \\
\hline Proteína (\%) & $10.069 \mathrm{a}$ & $9.605 c$ & $9.817 \mathrm{~b}$ & 9.83 & 0.146 \\
\hline Triptófano (\%) & $1.316 \mathrm{a}$ & $1.221 \mathrm{a}$ & $1.307 \mathrm{a}$ & 1.28 & 0.123 \\
\hline Lisina (\%) & $4.519 \mathrm{a}$ & $5.083 \mathrm{a}$ & $4.228 \mathrm{a}$ & 4.61 & 1.078 \\
\hline
\end{tabular}

DHS: diferencia significativa honesta (Tukey, $\mathrm{p} \leq 0.05$ ); variables con la misma letra estadísticamente son iguales.

química de híbridos y variedades de maíz, la variedad sintética VS-535 expresó 9.8 por ciento de proteína (Méndez-Montealvo et al. 2005).

De manera general, los porcentajes de triptófano y lisina de los genotipos presentaron promedios similares estadísticamente (Cuadro 4). Vivek et al. (2008) evaluaron la variedad de maíz V-537C en las localidades de Teotlalco y Chiautla, sin aplicar niveles floraciones y el rendimiento de grano (Sotomayor et al. 2017), entre otras características.

El porcentaje de proteína fue inferior estadísticamente al aplicar 100 unidades de nitrógeno, respecto a la aplicación de 150 unidades de nitrógeno. Este resultado indica que para obtener 10 por ciento de proteína, la dosis $150 \mathrm{~N}$ es la adecuada para la fertilización de los tres genotipos de maíz. La respuesta a 
la fertilización nitrogenada de los genotipos presentó valores inferiores estadísticamente en triptófano y lisina, cuando se aplicó 200 N. Los valores mayores fueron observados con $100 \mathrm{~N}$ (Cuadro 5). Estos resultados indicaron que al aplicar $200 \mathrm{~N}$ en una hectárea disminuyó el porcentaje de ambos aminoácidos esenciales presentes en el maíz.

Fósforo. El factor de variación fósforo (P) no afectó significativamente el rendimiento de grano de las variedades de maíz. La dosis $60 \mathrm{~kg}$ ha-1 P es la que debe recomendarse con los agricultores para la producción de grano de maíz en los ambientes estudiados, debido
$120 \mathrm{~kg} \mathrm{ha}^{-1} \mathrm{P}$. Cabe mencionar que el mayor porcentaje de proteína (10.0\%) fue observado con la dosis 60 y 120 $\mathrm{kg} \mathrm{ha}^{-1}$ de P; resultados similares fueron reportados por Zepeda-Bautista et al. (2009), al evaluar híbridos de cruza simple de maíz.

El mayor porcentaje de triptófano se observó con la dosis $90 \mathrm{~kg}$ ha- $1 \mathrm{P}$, mientras que 60 y $120 \mathrm{~kg}$ ha- 1 $\mathrm{P}$ exhibieron porcentajes inferiores estadísticamente. El menor porcentaje de lisina fue observado con 90 $\mathrm{kg}$ ha-1 P y estadísticamente fue inferior respecto a la dosis de $60 \mathrm{~kg}$ ha-1 P (Cuadro 6). Zepeda-Bautista et al. (2009) reportan resultados similares en híbridos

Cuadro 5. Efecto de tres dosis de nitrógeno, valorado en cuatro caracteres de maíz, considerando tres genotipos de maíz evaluados en dos localidades de Guerrero, México.

\begin{tabular}{llllll}
\hline & \multicolumn{5}{c}{ Nitrógeno (N) } \\
\cline { 2 - 4 } Variables & 100 & 150 & 200 & Media & DSH (0.05) \\
\hline Rendimiento de grano $\left(\mathrm{t} \mathrm{ha}^{-1}\right)$ & $5.028 \mathrm{~b}$ & $5.348 \mathrm{~b}$ & $5.839 \mathrm{a}$ & 5.41 & 0.480 \\
Proteína (\%) & $9.511 \mathrm{~b}$ & $10.025 \mathrm{a}$ & $9.955 \mathrm{a}$ & 9.83 & 0.146 \\
Triptófano (\%) & $1.419 \mathrm{a}$ & $1.229 \mathrm{~b}$ & $1.195 \mathrm{~b}$ & 1.28 & 0.123 \\
Lisina (\%) & $5.313 \mathrm{a}$ & $4.488 \mathrm{ab}$ & $4.031 \mathrm{~b}$ & 4.61 & 1.079 \\
\hline
\end{tabular}

DSH: diferencia significativa honesta (Tukey $=\alpha=\mathrm{p} \leq 0.05$ ); variables con la misma letra estadísticamente son iguales.

a que las características bioquímicas y la dosis aplicada de fertilizante exhibieron una respuesta aceptable en los genotipos de maíz. Las dosis 120 y 60 kg ha- 1 $\mathrm{P}$ produjeron similar rendimiento de grano, $\mathrm{y}$, al aumentar la cantidad de fertilizante, disminuyó la producción de grano (Cuadro 6).

Manjarrez et al. (2014) reportaron rendimiento de grano promedio de $4.67 \mathrm{t}$ ha- 1 al evaluar maíces de alta calidad de proteína, mientras que, en el presente estudio, el rendimiento de grano fue $809 \mathrm{~kg}$ mayor al aplicar $60 \mathrm{~kg}$ de fósforo. La dosis $90 \mathrm{~kg} \mathrm{ha}^{-1} \mathrm{P}$ exhibió menor porcentaje de proteína respecto a la dosis $60 \mathrm{P} \mathrm{y}$ de cruza simple de maíz fertilizados con $60 \mathrm{~kg}$ ha- 1 P. Estos autores informaron aumento en proteína y disminución significativa de triptófano. Este resultado indica que las variedades de maíz asimilaron el fertilizante de manera diferencial debido a sus características genéticas particulares, por lo que difirieron en el porcentaje de proteína, triptófano y lisina concentrado en el grano.

La combinación de $100 \mathrm{~N}+90 \mathrm{~kg}$ ha-1 P presentó mayor porcentaje de triptófano, y la combinación 100 N+60 P mostró mayor porcentaje de lisina. Los resultados indican que, al aplicar dosis mayores de

Cuadro 6. Efecto de tres dosis de fósforo, valorado en cuatro caracteres de maíz, considerando tres genotipos de maíz evaluados en dos localidades de Guerrero, México.

\begin{tabular}{llllll}
\hline & \multicolumn{3}{c}{ Fósforo (P) } & \\
\cline { 2 - 4 } Variables & 60 & 90 & 120 & Media & DSH \\
\hline Rendimiento de grano & $5.479 \mathrm{a}$ & $5.535 \mathrm{a}$ & $5.202 \mathrm{a}$ & 5.41 & 0.480 \\
Proteína & $10.029 \mathrm{a}$ & $9.370 \mathrm{~b}$ & $10.091 \mathrm{a}$ & 9.83 & 0.146 \\
Triptófano & $1.254 \mathrm{~b}$ & $1.377 \mathrm{a}$ & $1.213 \mathrm{~b}$ & 1.28 & 0.123 \\
Lisina & $5.125 \mathrm{a}$ & $4.062 \mathrm{~b}$ & $4.644 \mathrm{ab}$ & 4.61 & 1.079 \\
\hline
\end{tabular}


$100 \mathrm{~N}$ y $90 \mathrm{~kg}$ ha-1 P ha-1, disminuye el porcentaje de ambos aminoácidos esenciales presentes en las variedades de maíz.

\section{Conclusiones}

En ambas localidades, los caracteres medidos en los genotipos de maíz fueron semejantes, a excepción del porcentaje de proteína. La combinación de nitrógeno y fósforo influyó en el rendimiento de grano de las variedades VS-535 y V-537C. Las variedades VS-535 y A-7573 alcanzaron mayor porcentaje de proteína con la combinación $150 \mathrm{~N}+60$ P. La combinación 100 $\mathrm{N}+90$ P contribuyó en el porcentaje de triptófano en los genotipos VS-535 y A-7573. Las variedades A-7573 y V-537C interaccionaron con la combinación $100 \mathrm{~N}+60$ $P$ para producir mayor porcentaje de lisina. Se requiere una combinación específica de nitrógeno y fósforo para obtener buena respuesta y lograr la cantidad deseable en el contenido de proteína, triptófano y lisina en maíz. 


\section{Literatura Citada}

Adediran JA, Banjoko VA. 1995. Response of maize to nitrogen, phosphorus and potassium fertilizers in the savanna zone of Nigeria. Communications in Soil Science and Plant Analysis 26: 593-606. https://doi. org/10.1080/00103629509369320

Agama-Acevedo E, Juárez-García E, EvangelistaLozano S, Rosales-Reynoso OL, Bello-Pérez LA. 2013. Características del almidón de maíz y relación con las enzimas de su biosíntesis. Agrociencia 47: 1-12.

Andrés-Meza P, Sierra-Macías M, Mejía-Contreras JA, Molina-Galán J, Espinosa-Calderón A, GómezMontiel NO, Valdivia-Bernal R. 2014. Genotypeenvironment interaction in tropical maize varieties developed for the tropical region of Veracruz, México. Interciencia 39: 180-184.

Barrios M, Basso C. 2018. Efecto de la fertilización nitrogenada sobre componentes de rendimiento y calidad nutricional del grano de seis híbridos de maíz. Bioagro 30: 38-48.

Beres BL, McKenzie RH, Dowbenko RE, Badea CV, Spaner DM. 2012. Does handling physically alter the coating integrity of ESN urea fertilizer? Agronomy Journal 104: 1149-1159. https://doi.org/10.2134/ agronj2012.0044

Ciampitti IA, Vyn TJ. 2013. Grain nitrogen source changes over time in maize: A Review. Crop Science 53: 366-377. https://doi.org/10.2135/cropsci2012.07.0439

Ciampitti IA, Zhang H, Friedemann P, Vyn TJ. 2012. Potential physiological frameworks for mid-season field phenotyping of final plant $\mathrm{N}$ uptake, Nitrogen use efficiency, and grain yield in maize. Crop Science 52: 2728-2742. https://doi.org/10.2135/ cropsci2012.05.0305

Chura J, Mendoza-Cortez JW, de la Cruz JC. 2019. Dosis y fraccionamiento de nitrogeno en dos densidades de siembra del maíz amarillo duro. Scientia Agropecuaria 10: 241-248. https://doi.org/10.17268/ sci.agropecu.2019.02.09

Crow JF, Kermicle J. 2002. Oliver Nelson and quality protein maize. Genetics 160: 819-821.

Ding L, Wang KJ, Jiang GM, Biswas DK, Xu H, Li LF, Li YH. 2005. Effects of nitrogen deficiency on photosynthetic traits of maize hybrids released in different years. Annals of Botany 96: 925-930. https://doi. org/10.1093/aob/mci244
García E. 2004. Modificaciones al Sistema de Clasificación Climática de Köppen. Universidad Nacional Autónoma de México. Distrito Federal, México.

Gómez NO, Sierra M, Cantú MA, Rodríguez FA, Manjarrez M, González M, Espinosa A, Betanzos E, Córdova H, Caballero F, Turrent A, García A, Ramírez G, Sandoval A, Coutiño B, Cervantes E, Reyes C, Nava L. 2003. V-537C y V-538C, nuevas variedades de maíz con alta calidad de proteína para el trópico mexicano. Revista Fitotecnia Mexicana 26: 213-214.

Gómez NO, Palemón AF, Reyes GG, Hernández GCA, Cantú AMA, Juárez LP, Ascencio AA. 2016. Rendimiento de grano y características fenotípicas de maíz: efecto de ambiente y dosis de fertilización. Revista Mexicana de Ciencias Agrícolas 7: 1801-1813.

Haque MM, Hamid A, Bhuiyan NI. 2001. Nutrient uptake and productivity as affected by nitrogen and potassium application levels in maize/sweet potato intercropping system. Korean Journal of Crop Science 46: 1-5.

Iglesias S, Alegre J, Salas C, Egüez J. 2018. El rendimiento de maíz (Zea mays L.) mejora con el uso del biochar de eucalipto. Scientia Agropecuaria 9: 25-32. https://doi. org/10.17268/sci.agropecu.2018.01.03

Khaliq T, Ahmad A, Hussain A, Ali MA. 2009. Maize hybrids response to nitrogen rates at multiple locations in semiarid environment. Pakistan Journal of Botany 41: 207-224.

Kovács P, Scoyoc GEV, Doerge TA, Camberato JJ, Vyn TJ. 2014. Pre-plant anhydrous ammonia placement consequences on no-till versus conventional-till maize growth and nitrogen responses. Agronomy Journal 106: 634-644. https://doi.org/10.2134/agronj2013.0356

Liu W-Z, Zhang X-C. 2007. Optimizing water and fertilizer input using an elasticity index: A case study with maize in the loess plateau of China. Field Crops Research 100: 302-310. https://doi.org/10.1016/j.fcr.2006.08.005

Majnooni-Heris A, Zand-Parsa S, Reza-Sepaskhah AR, Kamgar-Haghighi AA, Yasrebi J. 2011. Modification and validation of maize simulation model (MSM) at different applied water and nitrogen levels under furrow irrigation. Archives of Agronomy and Soil Science 57: 401-420. https://doi.org/10.1080/03650340903512553

Manjarrez M, Palemón F, Gómez NO, Espinosa A, Rodríguez AS, Damián A, Hernández E, Cruz B. 2014. Aptitud combinatoria general y específica de maíces normales y de alta calidad de proteína. Revista Mexicana de Ciencias Agrícolas 5: 1261-1273. 
Masood T, Gul R, Munsif F, Jalal F, Hussain Z, Noreen N, Khan H, Din N, Khan H. 2011. Effect of different phosphorus levels on the yield and yield components of maize. Sarhad Journal of Agriculture 27: 167-170.

Méndez-Montealvo G, Solorza-Feria J, Velázquez-del Valle M, Gómez-Montiel N, Paredes-López O, BelloPérez LA. 2005. Composición química y caracterización calorimétrica de híbridos y variedades de maíz cultivadas en México. Agrociencia 39: 267-274.

Menkir A. 2008. Genetic variation of grain mineral content in tropical-adapted maize inbred lines. Food Chemistry 110: 454-464. https://doi.org/10.1016/j. foodchem.2008.02.025

Mertz ET, Bates LS, Nelson OE. 1964. Mutant gene that changes protein composition and increases lysine content of maize endosperm. Science 145: 279-280. https://doi.org/10.1126/science.145.3629.279

Mukhtar T, Arif A, Hussain S, Tariq M, Mehmood K. 2011. Effect of different rates of nitrogen and phosphorus fertilizers on growth and yield of maize. Journal Agricultural Research 49: 333-339.

Ortiz-Torres E, López PA, Gil-Muñoz A, GuerreroRodríguez JD, López-Sánchez $\mathrm{H}$, Taboada-Gaytán OR, Hernández-Guzmán JA, Valadez-Ramírez M. 2013. Rendimiento y calidad de elote en poblaciones nativas de maíz de Tehuacán, Puebla. Revista Chapingo Serie Horticultura 19(2): 225-238. https:// doi.org/10.5154/r.rchsh.2012.02.006

Owens BF, Lipka AE, Magallanes-Lundback M, Tiede T, Diepenbrock CH, Kandianis CB, Kim E, Cepela J, Mateos-Hernández M, Buell CR, Buckler ES, DellaPenna D, Gore MA, Rocheford T. 2014. A foundation for provitamin A biofortification of maize: genome-wide association and genomic prediction models of carotenoid levels. Genetics 198: 1699-1716. https://doi.org/10.1534/genetics.114.169979

Palemón-Alberto F, Gómez MNO, Castillo GF, Ramírez VP, Molina GJD, Miranda CS. 2011. Cruzas Intervarietales de maíz para la región semicálida de Guerrero, México. Revista Mexicana de Ciencias Agrícolas 2: 745-757.

Palemón-Alberto F, Gómez-Montiel NO, CastilloGonzález F, Ramírez-Vallejo P, Molina-Galán JD, Miranda-Colín S. 2012a. Estabilidad de cruzas intervarietales de maíz (Zea mays L.) para la región semicálida de Guerrero. Agrociencia 46: 133-145.

Palemón-Alberto F, Gómez MNO, Castillo GF, Ramírez
VP, Molina GJD, Miranda CS. 2012b. Potencial productivo de cruzas intervarietales de maíz en la región semicálida de Guerrero. Revista Mexicana de Ciencias Agrícolas 3: 157-171.

Palemón-Alberto F, Gómez-Montiel NO, Reyes-García G, Vargas-Álvarez D, Damián-Nava A, Cruz-Lagunas B, Hernández-Galeno CA, Hernández-Castro E. 2016. Rendimiento de grano de maíces (Zea mays L.) sembrados en la Costa Chica de Guerrero, México. Agro Productividad 9: 3-7.

Palemón-Alberto F, Gómez-Montiel NO, Reyes-García G, Vargas-Álvarez D, Damián-Nava A, Hernández-Castro E, Juárez-López P, Cruz-Lagunas B. 2017. Rendimiento de maíces cultivados en la región Tierra Caliente, Guerrero, México. Acta Agrícola y Pecuaria 3: 1-7.

Pillay K, Siwela M, Derera J, Veldman FJ. 2014. Provitamin A carotenoids in biofortified maize and their retention during processing and preparation of South African maize foods. Journal of Food Science and Technology 51: 634-644. https://doi.org/10.1007/s13197-011-0559-x

Sánchez MA, Aguilar CU, Valenzuela N, Joaquín BM, Sánchez C, Jiménez MC, Villanueva C. 2013. Rendimiento en forraje de maíces del trópico húmedo de México en respuesta a densidades de siembra. Revista Mexicana de Ciencias Pecuarias 4: 271-288.

Sotomayor R, Chura J, Calderón C, Sevilla R, Blas R. 2017. Fuentes y dosis de nitrógeno en la productividad de maíz amarillo duro bajo dos sistemas de siembra. Anales Científicos 78: 232-240. https://doi. org/10.21704/ac.v78i2.1061

Torres-Morales B, Coutiño-Estrada B, Muñoz-Orozco A, Santacruz-Varela A, Mejía-Contreras A, Serna-Saldivar SO, García-Lara S, Palacios-Rojas N. 2010. Selección para contenido de aceite en el grano de variedades de maíz de la raza Comiteco de Chiapas, México. Agrociencia 44: 679-689.

Uribelarrea M, Below FE, Moose SP. 2004. Grain composition and productivity of maize hybrids derived from the Illinois protein strains in response to variable nitrogen supply. Crop Science 44: 1593-1600. https://doi. org/10.2135/cropsci2004.1593

Vera-Guzmán AM, Chávez-Servia JL, Carrillo-Rodríguez JC. 2012. Proteína, lisina y triptófano en poblaciones nativas de maíz Mixteco. Revista Fitotecnia Mexicana 35 (Núm. Especial): 7-13.

Vivek BS, Krivanek AF, Palacios-Rojas N, Twumasi-Afriyie S, Diallo AO. 2008. Mejoramiento de Maíz con Calidad 
de Proteína (QPM). Protocolos para Generar Variedades QPM. Centro Internacional de Mejoramiento de Maíz y Trigo. Distrito Federal, México.

Xie G, Li Z, Ran Q, Wang H, Zhang J. 2018. Over-expression of mutated $Z m D A 1$ or $Z m D A R 1$ gene improves maize kernel yield by enhancing starch synthesis. Plant Biotechnology Journal 16: 234-244. https://doi. org/10.1111/pbi.12763

Zepeda-Bautista R, Carballo-Carballo A, Muñoz-Orozco A, Mejía-Contreras JA, Figueroa-Sandoval B, GonzálezCossio FV, Hernández-Aguilar C. 2009. Proteína, triptófano y componentes estructurales del grano en híbridos de maíz (Zea mays L.) producidos bajo fertirrigación. Agrociencia 43: 143-152.

Zhang W, Yang W, Wang M, Wang W, Zeng G, Chen Z, Cai Y. 2013. Increasing lysine content of waxy maize through introgression of Opaque-2 and Opaque-16 genes using molecular assisted and biochemical development. PloS ONE 8(2): e56227. https://doi.org/10.1371/ journal.pone. 0056227 